\title{
Associations between differing magnitudes of inter-limb asymmetry and linear and change of direction speed performance in male youth soccer players
}

\author{
Ali Işın ${ }^{1}$, Eren Akdağ², Emel Çetin Özdoğan ${ }^{1}$, Chris Bishop $^{3}$ \\ ${ }^{1}$ Department of Coaching Education, Faculty of Sport Sciences, Akdeniz University, Antalya, Turkey; ${ }^{2}$ Department of \\ Coaching Education, Faculty of Sport Sciences, Alanya Alaaddin Keykubat University, Antalya, Turkey; ${ }^{3}$ London Sport \\ Institute, School of Science and Technology, Middlesex University, London, UK
}

\begin{abstract}
Study aim: This study examines the relationship between different magnitudes of asymmetry and their effects on speed performance.

Material and methods: Forty-two sub-elite male youth soccer players performed a 30-m sprint, change of direction, single leg countermovement jump and single leg hop. Subjects were divided into groups with vertical and horizontal asymmetry, and both groups were then divided into three groups according to magnitudes of inter-limb asymmetry $(<5 \%, 5-10 \%$, and $>10 \%)$.

Results: The results showed no significant correlation between different jump asymmetry magnitudes and the mentioned outcomes of speed performance ( $\mathrm{p}>0.05)$. In addition, larger asymmetries resulted in faster linear speed, even if small differences ( $\mathrm{g}$ range $=0.00$ to $0.57 ; \mathrm{p}>0.05$ ). But this was not similar for change of direction speed $(\mathrm{g}$ range $=-0.42$ to $0.34 ; \mathrm{p}>0.05$ ).

Conclusions: There are inconsistent findings for the effects of inter-limb asymmetries on speed performance. The results of the present study indicate that the magnitude of asymmetry had no meaningful association with independent measures of performance in soccer players. Therefore, it seems more likely to explain the effects of individual asymmetries on performance rather than the idea that asymmetry negatively affects performance.
\end{abstract}

Keywords: Between-limb differences - Asymmetry assessment - Speed - Vertical - Horizontal

\section{Introduction}

The concept of inter-limb asymmetry-refers to the performance or function of one limb relative to another and has been a popular research topic in recent years [20]. Several classifications have been reported to measure these limb differences, including dominant and non-dominant [28], stronger and weaker [30], right and left [1], unilateral and bilateral [6], and injured and uninjured limbs [27]. Typically, studies have focused on the extent of inter-limb differences in various testing methods, such as isokinetic dynamometer [29, 31], isometric squat [7] or mid-thigh pulls [14], back squatting [30], and jumping [3, 8, 24].

Due to the nature of soccer, players frequently repeat unilateral and asymmetrical movements such as changes of direction and kicks [26]. In a single match, elite soccer players may perform 1200-1400 changes of direction while covering a total distance of 10-11 km [9]. In addition, players frequently perform activities such as passing, shooting, and dribbling. During all these activities, one limb is more dominant than the other and therefore, asymmetry is expected to be prevalent and indeed common in soccer [26]. In fact, previous studies have obtained high asymmetry scores (e.g., $>15 \%$ ) in professional $[4,26]$, amateur [33] and recreational soccer players [22].

Some studies have shown meaningful correlations between jump asymmetry and athletic performance [8, 10]. Bishop et al. [8] reported that drop jump asymmetries in female soccer players were positively correlated with $10 \mathrm{~m}$ $(\mathrm{r}=0.52), 30 \mathrm{~m}(\mathrm{r}=0.58)$, and change of direction (COD) (right; $r=0.52$ and left; $r=0.66$ ). however, it is important to note that the positive correlation was indicative of larger asymmetries being associated with slower linear and COD speed times. There is also evidence that high asymmetries can negatively affect performance in COD, vertical jump 
[35], and kicking accuracy [17]. In contrast, some studies have shown no associations between inter-limb differences and independent measures of physical performance [22, 23]. Lockie et al. [22] even reported that higher vertical jump asymmetries (22.1\%) had better 5-, 10-, and 20-m performances compared to low asymmetries $(4.5 \%)$. These conflicting findings highlight the need for further research in this area.

Although most studies have attempted to explain the relationship between asymmetry and performance, there are also recent studies that specifically address the effects of differing magnitudes of asymmetry on performance [26]. Dos'Santos et al. [13] and Lockie et al. [22] assessed asymmetry size as lesser and greater in two groups to examine the effects of asymmetry magnitude on performance. In these two studies, no meaningful differences in sprint or COD performance were found between groups $[13,22]$. Interestingly though, the group with greater asymmetry did perform better than the group with less asymmetry on some performance measures. However, the evidence obtained from the results of this study suggests that a dichotomous comparison is insufficient for the relationship between asymmetry size and performance. In addition, previous research has shown a lack of data on the relationship between different magnitudes of asymmetry and athletic performance. Therefore, less is known about the relationship between different magnitudes of asymmetry and athletic performance [26]. Furthermore, different magnitudes of asymmetry are expected in soccer players [4], and the evaluation of asymmetry magnitudes obtained from jump tests provides opportunities to investigate whether asymmetry magnitudes influence performance in soccer players. Hence, the primary aim of this study was to classify inter-limb asymmetries produced from single leg countermovement jump (SLCMJ) and single leg hop (SLHOP) test into low, medium and high groups according to magnitude of asymmetry, and to investigate the relationship between different magnitudes of asymmetry and speed performance. Thus, it can contribute to explain the possible effects of the magnitudes of asymmetry on speed performance.

\section{Material and methods}

\section{Experimental approach to the problem}

This study focused on the relationship between differing magnitudes of inter-limb asymmetry and linear and change of direction speed performance in male youth soccer players. Players performed a COD speed, sprint (10, 20 and $30 \mathrm{~m}$.), SLCMJ and SLHOP. Vertical asymmetry was calculated using the jump height obtained from the SLCMJ and horizontal asymmetry was calculated using the distance obtained from the SLHOP. Players were asked not to perform in any physical activity the day before the measurement day. After a familiarization session with all participants, all data were measured during a single session and in the same order. Tests were measured following a standard 10-min warm-up protocol consisting of stretching exercises such as standing hamstring stretch, knee to chest stretch, standing quadriceps stretch, calf stretch, and multi-planar lunges. A 5-min rest period was included between each task (SLCMJ, SLHOP, 5-0-5 COD and 30-m sprint test).

\section{Subjects}

Forty two young sub-elite male soccer players (age: $15.19 \pm 1.04$ years; height: $167.57 \pm 10.00 \mathrm{~cm}$; body weight: $56.97 \pm 11.78 \mathrm{~kg}$; BMI: $20.08 \pm 2.55 \mathrm{~kg} \cdot \mathrm{m}^{-2}$ ) from the same team volunteered to participate in the study. The players are divided into vertical and horizontal asymmetry groups, and both groups were then classified into three groups according to magnitudes of inter-limb asymmetry $(<5 \%, 5-10 \%$, and $>10 \%)$. asymmetry). The study was approved by the Akdeniz University Clinical Research Ethics Committee (2019-596) and was conducted in accordance with the Helsinki Declaration. All participants were informed of the research procedure and informed consent forms were obtained from both participants and their families.

\section{Procedures}

Single leg countermovement jump (SLCMJ). Subjects were asked to stand in an upright position, hands on hips, with feet positioned hip width apart and then lifted one leg off the floor. Subjects then moved vertically, jumping as high as possible. If the jumping leg was not fully extended and hands were not fixed on the hips, the test was repeated after a 60 -second rest period. [8]. SLCMJ was measured using Optojump (Microgate, Bolzano, Italy). Optojump has been shown to be a valid and reliable method for estimating jump height [16]. The SLCMJ was tested twice on each leg, separated by a 30 -second rest period. The highest jump height in both legs was evaluated for data analysis [10].

Single leg hop jump (SLHOP). Subjects were asked to stand on the test foot with their hands on the hips and toes behind the starting line, and then hop as far forward as possible and land on the same leg. If subjects were unable to hold their position for 2 seconds upon landing, the test was repeated after a 60 second rest. The distance between the starting line and the point where the subject's heel hit touched the floor was measured by standard measuring tape and recorded in centimetres $(\mathrm{cm})$ [8]. The SLHOP was tested twice on each leg, separated by a 30 -second rest period. The longest jump distance in both legs was evaluated for data analysis [10].

Chance of direction (COD). The 505 test was applied to evaluate the subjects' change of direction performance. The 
505 test consists of a $10 \mathrm{~m}$ forward and $5 \mathrm{~m}$ turn to run from the start line. The time starts in the photocell stopwatch, which places it $10 \mathrm{~m}$ ahead of the starting line, the subject goes $5 \mathrm{~m}$, turns $180^{\circ}$ with the subject's right or left foot, turns to the same point and the time is recorded. The COD was tested twice on the leg that the players determined as the dominant, separated by a 90 -second rest period. The fastest trial was evaluated for data analysis [10] (Fig. 1A).

30-m sprint test. Subjects were asked to stand $30 \mathrm{~cm}$ behind the starting line and run forward at a predetermined distance of $30 \mathrm{~m}$. Photocells (Witty gate, Microgate, Bolzano, Italy) were positioned at $0,10,20$ and $30 \mathrm{~m}$ (Fig. 1B).

The vertical and horizontal asymmetry calculation. Vertical asymmetry was calculated from the jump height in the SLCMJ test. Horizontal asymmetry was calculated from the distance in the SLHOP test. The formula below was used to determine the percentage of both asymmetries [6]. The maximum value indicates the longest or highest jump performances of the players, the minimum value the shortest or lowest jump values.

$$
\text { Asymmetry }=\frac{\max \text { value }(\mathrm{cm})-\min \text { value }(\mathrm{cm})}{\max \text { value }(\mathrm{cm})} \cdot 100 \%
$$

\section{Statistical analyses}

All data were presented as mean \pm standard deviation (mean \pm Std.). Shapiro-Wilk test was used to determine if the data were normally distributed. Intraclass correlation coefficient (ICC) with absolute agreement including

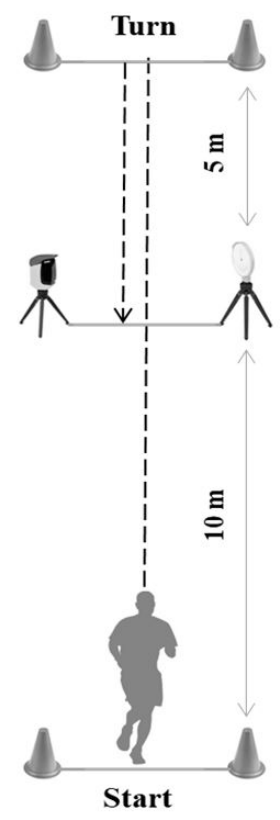

A

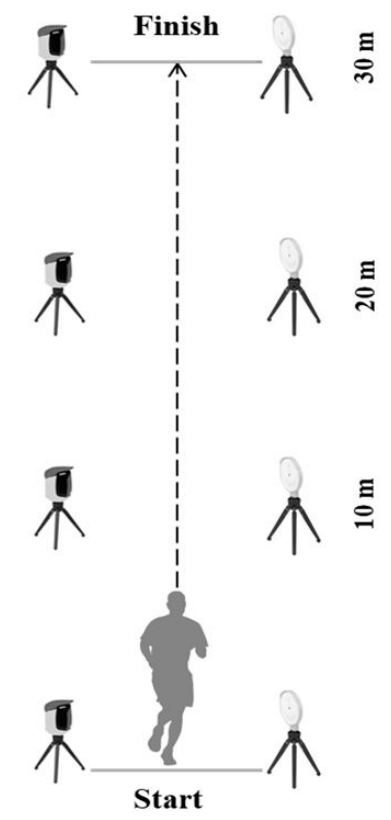

B
Figure. 1. A. Chance of direction (5-0-5 COD) B. Sprint $(10,20$ and $30 \mathrm{~m})$
$95 \%$ confidence intervals ( $95 \% \mathrm{CI})$ and the coefficient of variance $(\mathrm{CV})$ were used to analyse within-session reliability of test measures. A one-way analysis-of variance (ANOVA) with the Bonferroni post hoc was applied to determine if any statistical differences between groups were evident. Hedges $g$ effect sizes with $95 \%$ confidence intervals were also computed to provide an understanding of practical differences and were interpreted as suggested by Hopkins et al. [19]: trivial $<0.20$, small $=0.20-0.59$, $\bmod -$ erate $=0.60-1.19$, large $=1.20-1.99$ and very large $\geq 2.00$ ). ICC was evaluated as reported by Koo and $\mathrm{Li}$ [21] where: $>0.9=$ excellent, $0.75-0.9=$ good, $0.5-0.74=$ moderate and $<0.5=$ poor. If $\mathrm{CV}$ values were $<10 \%$, it was deemed acceptable [12]. Kappa coefficients were calculated to determine levels of agreement in the direction of asymmetry between jump tests [11] and interpreted as proposed by Viera and Garrett [32] where: $<0=$ poor, $0-0.2=$ slight, $0.21-0.4=$ fair, $0.41-0.6=$ moderate, $0.61-0.8=$ substantial, $0.81-0.99=$ nearly perfect and $1=$ perfect. Pearson correlation was used to analyse the relationship between leg asymmetries (vertical and horizontal) and sprint (10, 20 and 30m.) and COD speed. SPSS (IBM 23v., Armonk, NY, USA) and Excel (2020v., Microsoft, Redmond, WA) were used for statistical analysis and statistical significance was accepted as $\mathrm{p}<0.05$ for all data.

\section{Results}

The ICC values were between 0.86 and 0.99 for all tests and the CV values were between $0.94 \%$ and $4.16 \%$ for all tests.

The vertical asymmetry (14.61\%) produced from the SLCMJ (right: $11.29 \mathrm{~cm}$; left: $11.08 \mathrm{~cm}$ ) was greater than the horizontal asymmetry $(6.04 \%)$ produced from SLHOP (right:129.90 cm; left:127.10 cm). Table 1 shows jump scores and the magnitude of asymmetry for each of the 3 asymmetry groups (i.e $<5 \%, 5-10 \%$ and $>10 \%$ ) in both the SLCMJ and SLHOP tests. There were no significant differences in jump scores for either test, relative to different magnitudes of asymmetry ( $g$ range $=-0.64$ to 0.12 ; $\mathrm{p}>0.05)$. However, significant $(\mathrm{p}<0.05)$ and very large differences $(g>2.0)$ between mean asymmetry for the three groups (Table 1).

Table 2 demonstrates sprint (10-, 20-, and 30-m) and COD speed performance for each of the 3 asymmetry groups (i.e., $<5 \%, 5-10 \%$ and $>10 \%$ ) in both the SLCMJ and SLHOP tests. There were no significant differences in sprint (10-, 20-, and 30-m) and COD performance for either test, relative to different magnitudes of asymmetry ( $\mathrm{g}$ range $=-0.43$ to $0.57 ; \mathrm{p}>0.05)$ (Table 2$)$.

Table 3 shows correlations between leg asymmetries (vertical and horizontal) and 30-m sprint (10, 20 and 30m) and COD performance. No significant relationships were 

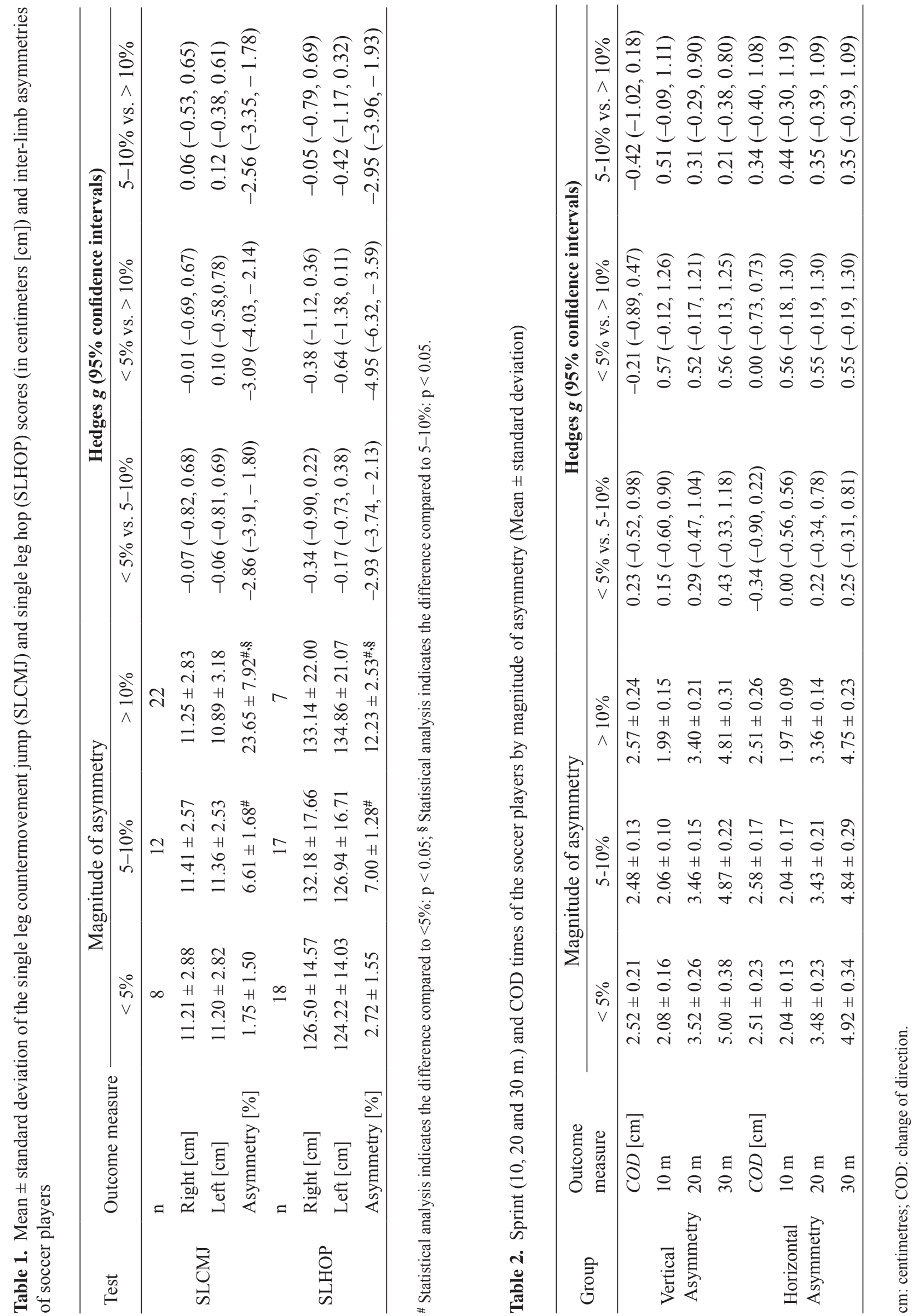
Table 3. Correlations between inter-limb asymmetries (vertical and horizontal) and sprint (10-, 20- and 30-m.) and COD performance

\begin{tabular}{lcccc}
\hline \multirow{2}{*}{ Asymmetry } & COD $[\mathrm{sec}]$ & \multicolumn{3}{c}{30 -m sprint } \\
\cline { 3 - 5 } & & $10 \mathrm{~m}[\mathrm{sec}]$ & $20 \mathrm{~m}[\mathrm{sec}]$ & $30 \mathrm{~m}[\mathrm{sec}]$ \\
\hline Vertical asymmetry & 0.05 & -0.11 & -0.02 & 0.01 \\
Horizontal asymmetry & -0.06 & -0.05 & -0.13 & -0.17 \\
\hline
\end{tabular}

Vertical asymmetry: calculated from SLCMJ scores; Horizontal asymmetry: calculated from SLHOP scores; COD: change of direction

found between both vertical and horizontal asymmetry and linear or COD performance (Table 3).

Figure 2 demonstrates soccer players' individual vertical and horizontal asymmetry values, and has been included owing to the large within-group variation between tests. There were 51 (23 for SLCMJ and 28 for SLHOP) positive (in favour of the right leg) and 33 (19 for SLCMJ and 14 for SLHOP) negative (in favour of the left leg) asymmetries, which resulted in slight levels of agreement and a Kappa coefficient of 0.10 .

\section{Discussion}

This study examined the effect of vertical and horizontal asymmetries from the SLCMJ and SLHOP tests on the 30-m sprint and COD in male youth soccer players and focused on whether performance was affected by the magnitudes of inter-limb asymmetry. The results showed no significant differences between different magnitudes of jump asymmetry and the aforementioned speed performance outcomes. On the contrary, although statistically significant differences were not found, groups with higher asymmetry mainly showed better sprint speed. Moreover, no significant correlation was found between vertical and horizontal asymmetries and speed performance (sprint and COD).

Firstly, we found that the vertical asymmetry (14.61\%) produced from the SLCMJ was greater than the horizontal asymmetry produced from SLHOP (6.04\%). Lockie et al. [22] that SLCMJ asymmetries of male recreational team athletes aged 18 years and older were higher than HOP asymmetries. The study conducted on female soccer

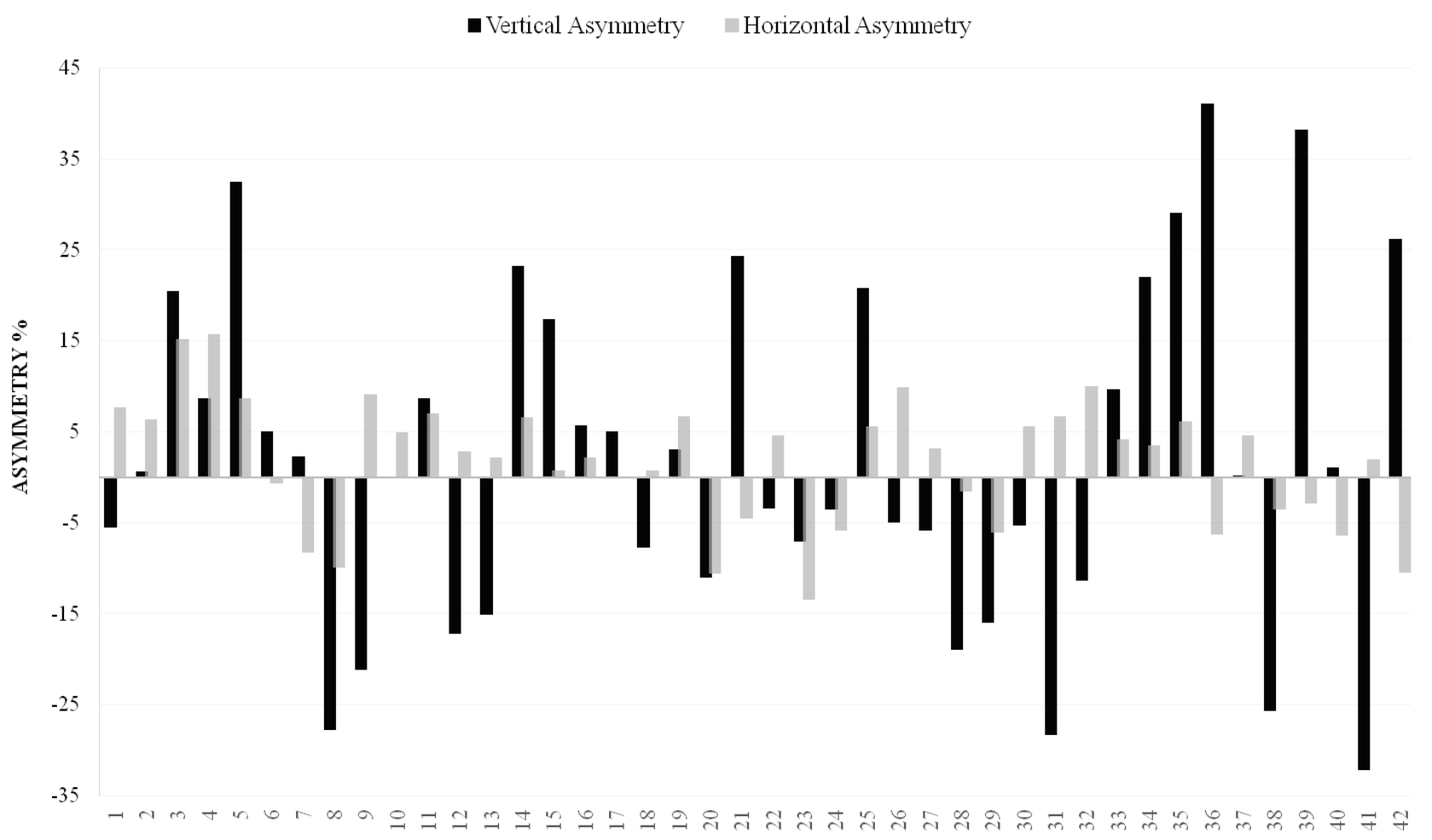

Figure 2. Soccer players' individual vertical and horizontal asymmetry percentages (negative values indicate that raw scores on the left limb are greater) 
players found that elite youth soccer players' SLCMJ asymmetries were significantly higher compared to other jump asymmetries [8]. Although studies on different groups reported similar results, the underlying mechanism is not clear. Bishop et al. [8] suggested that children perform more horizontal hopping activities at an early age, resulting in lower horizontal asymmetries. Considering that soccer players also perform more horizontally oriented movements, their vertical asymmetries are likely to be greater. Furthermore, the direction of the force generated and the measured performance during the vertical jump is in the same direction, but not during a SLHOP (transversal) [2] . This could be the source of the large differences between vertical and horizontal asymmetry.

This study showed that the magnitudes of the asymmetry did not affect the raw jump scores on both the left and right sides. This was especially true for the SLCMJ (g range $=-0.07$ to $0.12 ; p>0.05$ ), whereas for SLHOP (g range $=-0.64$ to $-0.05 ; \mathrm{p}>0.05)$, larger asymmetries resulted in greater jump distance. When interpreting the findings of SLHOP asymmetry, those with $>10 \%$ asymmetry had a greater jump distance than those with $<5 \%$ and $5-10 \%$ asymmetry (for right limb $g=-0.38$ to -0.05 and for left limb $g=-0.64$ to -0.42 , respectively). Bell et al. [3], who divided the asymmetry into four groups as $0-5 \%, 5-10 \%, 10-15 \%$ and $>15 \%$ according to the magnitude of the asymmetry, reported a result similar to the results of our study. The group with $>15 \mathrm{CMJ}$ force asymmetry $(38.8 \mathrm{~cm}$; $95 \%$ CI: $31.5-46.1)$ had the higher jump height compared to the other groups $(0-5 \%=36.1 \mathrm{~cm}$; 95\% CI: $34.2-37.9,5-10 \%=34.8 \mathrm{~cm}$; $95 \%$ CI: $32.6-37.1$ and $10-15 \%=33.6 \mathrm{~cm} ; 95 \%$ CI: 30.2-37.0). However, the number of those with $>15$ asymmetry (n:7) was very small compared to the other groups $(0-5 \% \mathrm{n}: 88 ; 10-15 \% \mathrm{n}: 45$ and $10-15 \% \mathrm{n}: 27)$, which is a limitation that could affect the result. Nevertheless, it cannot be said that increased asymmetry necessarily negatively affects performance. Given these results, it seems more likely to explain the effects of individual asymmetries on performance than the idea that asymmetry negatively affects performance.

In the current study, no significant relationship was found between asymmetry magnitudes and sprint and COD speed $(p>0.05)$. Furthermore, larger asymmetries resulted in faster linear speed, although the differences were small ( $\mathrm{g}$ range $=0.00$ to $0.57 ; \mathrm{p}>0.05$ ). However, this was not similar for COD speed ( $\mathrm{g}$ range $=-0.42$ to 0.34; $p>0.05$ ). Dos'Santos et al. [15] reported that interlimb asymmetry had no affect COD performance. Similarly, there was no relationship between the asymmetries calculated from unilateral jump tests and multidirectional sprint performance [22], and no relationship was reported between vertical asymmetry produced from vertical jump test and COD [18]. In contrast to these results, Michailidis et al. [25] reported a moderate correlation $(r=0.540$, $\mathrm{p}<0.05$ ) between SLCMJ asymmetry and COD, and they found no correlation between COD and other jump asymmetries. Asymmetries can be thought to not affect COD performance due to athletes performing different techniques as a response to the power or strength imbalance between the limbs [22]. Furthermore, since players prefer the stronger leg during COD and the stronger leg compensates for the weaker leg [34], it is likely that asymmetries do not affect COD performance. Moreover, considering the vertical asymmetry scores, the best COD speed was in the $5-10 \%$ asymmetry group, while according to the horizontal asymmetry scores, the best COD speed was in the $5 \%$ and $10 \%$ asymmetry groups. Interestingly, those with $5-10 \%$ vertical asymmetries performed best, but those with $5-10 \%$ horizontal asymmetry did the worst. This result may lead to the idea that asymmetry may have a taskspecific effect on performance outcomes [4].

There was no significant correlation between vertical or horizontal asymmetry and linear or COD speed $(p>0.05)$ and this result was consistent with the research findings of Lockie et al. [22] and Loturco et al. [23]. In contrast, Bishop et al. [8] found a moderate correlation between asymmetry and 10-, 30-m and COD performance. These different results indicate that individual differences should be considered when explaining the relationship between asymmetry and performance [4]. We also applied the kappa coefficient statistic to determine levels of agreement in the direction of asymmetry between jump tests, and showed that only slight levels of agreement were evident between jump tests $(\mathrm{Kappa}=0.10)$. Simply put, if a player jumped further on their right leg in the SLHOP, it was not overly common for the right leg to perform best on the SLCMJ. For example, in Figure 2, athlete 36 was right limb dominant with a vertical asymmetry of $41 \%$, while the left limb was dominant with a horizontal asymmetry of $6.4 \%$, resulting in a $47.4 \%$ shift in asymmetry from right to left limb. This further highlights the task-specificity of asymmetry / limb dominance as reported in previous studies recently $[4,5]$.

In conclusion, the results of this research focusing on the relationship between different magnitudes of asymmetry and their effects on speed performance indicate that the magnitude of asymmetry had no meaningful association with independent measures of performance in soccer players.

Finally, this research has some limitations. All the soccer players participating in the study were members of the same soccer club. The training levels of this club may have influenced the results of this study. In addition, participants in the study were selected using the convenience sampling method. Future studies could use power analysis to determine the number of participants needed. Participants performed the COD by only one direction, which may have provided an advantage for athletes with 
directional dominance [15]. Thus, future research should inspect both directions in COD. Lastly, as the players had lower horizontal asymmetries, the number of subjects in the $>10 \%$ was relatively lower compared to in the other horizontal asymmetry groups. This may have affected the statistical analysis of the research results.

\section{Practical applications}

Asymmetry imbalances can occur in soccer players as a result of a variety of actions such as jumping, COD and kicking the ball. However, the results of the present study indicate that the magnitude of asymmetry had no meaningful association with independent measures of performance in soccer players. For this reason, practitioners and coaches should not prioritise measuring asymmetry. Rather, they should simply prioritise measuring the raw scores from the tests themselves, as opposed to any relative limb differences.

\section{Conflict of interest: Authors state no conflict of interest.}

\section{References}

1. Atkins S.J., Bentley I., Hurst H.T., Sinclair J.K., Hesketh C. (2016) The presence of bilateral imbalance of the lower limbs in elite youth soccer players of different ages. $J$. Strength Cond. Res., 30: 1007-1013.

2. Augustsson J., Thomee R., Linden C., Folkesson M., Tranberg R., Karlsson J. (2006) Single-leg hop testing following fatiguing exercise: reliability and biomechanical analysis. Scand. J. Med. Sci. Sports, 16: 111-120.

3. Bell D.R., Sanfilippo J.L., Binkley N., Heiderscheit B.C. (2014) Lean mass asymmetry influences force and power asymmetry during jumping in collegiate athletes. $J$. Strength Cond. Res., 28: 884.

4. Bishop C., Read P., Chavda S., Jarvis P., Brazier J., Bromley T., Turner A.N. (2020) Magnitude or Direction? Seasonal variation of interlimb asymmetry in elite academy soccer players. J. Strength Cond. Res. DOI: 10.1519/ JSC.0000000000003565.

5. Bishop C., Read P., Chavda S., Jarvis P., Turner A. (2019) Using unilateral strength, power and reactive strength tests to detect the magnitude and direction of asymmetry: a test-retest design. Sports, 7: 58.

6. Bishop C., Read P., Lake J., Chavda S., Turner A. (2018) Interlimb asymmetries: understanding how to calculate differences from bilateral and unilateral tests. Strength Cond. J., 40: 1-6.

7. Bishop C., Read P., Lake J., Loturco I., Dawes J., Madruga M., Romero-Rodrigues D., Chavda S., Turner A. (2021) Unilateral isometric squat: Test reliability, interlimb asymmetries, and relationships with limb dominance. J. Strength Cond. Res., 35: 144-151.
8. Bishop C., Read P., McCubbine J., Turner A. (2021) Vertical and horizontal asymmetries are related to slower sprinting and jump performance in elite youth female soccer players. J. Strength Cond. Res., 35: 56-63.

9. Bishop C., Read P., Stern D., Turner A.N. (2020) Effects of Soccer match-play on unilateral jumping and Interlimb asymmetry: a repeated measures design. J. Strength Cond. Res. 36: 193-200.

10. Bishop C., Turner A., Maloney S., Lake J., Loturco I., Bromley T., Read P. (2019) Drop jump asymmetry is associated with reduced sprint and change-of-direction speed performance in adult female soccer players. Sports, 7: 29 .

11. Cohen J. (1960) A coefficient of agreement for nominal scales. Educ. Psychol. Meas., 20: 37-46.

12. Cormack S.J., Newton R.U., McGuigan M.R., Doyle T.L. (2008). Reliability of measures obtained during single and repeated countermovement jumps. Int. J. Sports Physiol. Perform., 3: 131-144.

13. Dos' Santos T., Thomas C., Jones P.A., Comfort P. (2019) Assessing asymmetries in change of direction speed performance: Application of change of direction deficit. $J$. Strength Cond. Res., 33: 2953-2961.

14. Dos' Santos T., Thomas C., Jones P.A., Comfort P. (2017) Assessing Muscle-Strength Asymmetry via a UnilateralStance Isometric Midthigh Pull. Int. J. Sports Physiol. Perform., 12: 505-511.

15. Dos' Santos T., Thomas C., Jones P.A., Comfort P. (2017) Asymmetries in single and triple hop are not detrimental to change of direction speed. J. Trainol., 6: 35-41.

16. Glatthorn J.F., Gouge S., Nussbaumer S., Stauffacher S., Impellizzeri F.M., Maffiuletti N.A. (2011). Validity and reliability of Optojump photoelectric cells for estimating vertical jump height. J. Strength Cond. Res., 25: 556-560.

17. Hart N.H., Nimphius S., Spiteri T., Newton R.U. (2014). Leg strength and lean mass symmetry influences kicking performance in Australian Football. J. Sports Sci. Med., 13: 157.

18. Hoffman J.R., Ratamess N.A., Klatt M., Faigenbaum A.D., Kang J. (2007) Do bilateral power deficits influence direction-specific movement patterns? Res. Sports Med., 15: 125-132.

19. Hopkins W.G. (2010) Linear models and effect magnitudes for research, clinical and practical applications. Sportscience, 14: 49-59.

20. Keeley D.W., Plummer H.A., Oliver G.D. (2011) Predicting asymmetrical lower extremity strength deficits in college-aged men and women using common horizontal and vertical power field tests: A possible screening mechanism. J. Strength Cond. Res., 25: 1632-1637.

21. Koo T.K., Li M.Y. (2016) A guideline of selecting and reporting intraclass correlation coefficients for reliability research. J. Chiropr. Med., 15: 155-163. 
22. Lockie R.G., Callaghan S.J., Berry S.P., Cooke E.R., Jordan C.A., Luczo T.M., Jeffriess M.D. (2014) Relationship between unilateral jumping ability and asymmetry on multidirectional speed in team-sport athletes. J. Strength Cond. Res., 28: 3557-3566.

23. Loturco I., Pereira L.A., Kobal R., Abad C.C., Rosseti M., Carpes F.P., Bishop C. (2019) Do asymmetry scores influence speed and power performance in elite female soccer players? Biol. Sport., 36: 209.

24. Maloney S.J., Fletcher I.M., Richards J. (2016) A comparison of methods to determine bilateral asymmetries in vertical leg stiffness. J. Sports Sci., 34: 829-835.

25. Michailidis Y., Savvakis C., Pirounakis V., Mikikis D., Margonis K., Metaxas T. (2020) Association between jump asymmetry and reduced performance in the change of direction tests of youth soccer players. J. Phys. Educ. Sport., 20: 1362-1368.

26. Read P.J., McAuliffe S., Bishop C., Oliver J.L., GrahamSmith P., Farooq M.A. (2021) Asymmetry thresholds for common screening tests and their effects on jump performance in professional soccer players. J. Athl. Train., 56: 46-53.

27. Rohman E., Steubs J.T., Tompkins M. (2015) Changes in involved and uninvolved limb function during rehabilitation after anterior cruciate ligament reconstruction: implications for Limb Symmetry Index measures. Am. J. Sports Med., 43: 1391-1398.

28. Rouissi M., Chtara M., Owen A., Chaalali A., Chaouachi A., Gabbett T., Chamari K. (2016) Effect of leg dominance on change of direction ability amongst young elite soccer players. J. Sports Sci., 34: 542-548.

29. Ruas C.V., Brown L.E., Pinto R.S. (2015) Lower-extremity side-to-side strength asymmetry of professional soc- cer players according to playing position. Kinesiol. Int. J. Fundam. Appl. Kinesiol., 47: 188-192.

30. Sato K., Heise G.D. (2012) Influence of weight distribution asymmetry on the biomechanics of a barbell back squat. J. Strength Cond. Res., 26: 342-349.

31. Silva J.R.L.C., Detanico D., Pupo J.D., Freitas C.d.1.R. (2015) Bilateral asymmetry of knee and ankle isokinetic torque in soccer players u20 category. Rev. Bras. de Cineantropometria e Desempenho Hum., 17: 195-204.

32. Viera A.J., Garrett J.M. (2005) Understanding interobserver agreement: the kappa statistic. Fam. Med., 37: 360-363.

33. Yanci J., Camara J. (2016) Bilateral and unilateral vertical ground reaction forces and leg asymmetries in soccer players. Biol. Sport., 33: 179.

34. Yoshioka S., Nagano A., Hay D.C., Fukashiro S. (2010) The effect of bilateral asymmetry of muscle strength on jumping height of the countermovement jump: A computer simulation study. J. Sports Sci., 28: 209-218.

35. Young W.B., James R., Montgomery I. (2002) Is muscle power related to running speed with changes of direction? J. Sports Med. Phys. Fitness, 42: 282-288.

\section{Received 06.10.2021 \\ Accepted 22.11.2021}

(C) University of Physical Education, Warsaw, Poland

\section{Acknowledgments}

This study was supported by the Scientific Research Projects Coordination Unit of Akdeniz University, Turkey. Project Number: TSA-2019-4936. 